\title{
Knowledge Acquisition and Construction of a RDF-Ontology for Computer-Assisted Surgery
}

\author{
Niclas HAGEN ${ }^{\mathrm{a}, 1}$, Reinald KÜHLE ${ }^{\mathrm{b}}$, Frederic WEICHEL ${ }^{\mathrm{b}}$, Urs EISENMANN ${ }^{\mathrm{a}}$, \\ Petra KNAUP-GREGORI ${ }^{\mathrm{a}}$ and Christian FREUDLSPERGER ${ }^{\mathrm{b}}$ \\ anstitute of Medical Biometry and Informatics, University of Heidelberg, \\ Heidelberg, Germany \\ ${ }^{\mathrm{b}}$ Department of Oral and Maxillofacial Surgery, Heidelberg University Hospital, \\ Heidelberg, Germany
}

\begin{abstract}
The integration of surgical knowledge into virtual planning systems plays a key role in computer-assisted surgery. The knowledge is often implicitly contained in the implemented algorithms. However, a strict separation would be desirable for reasons of maintainability, reusability and readability. Along with the Department of Oral and Maxillofacial Surgery at Heidelberg University Hospital, we are working on the development of a virtual planning system for mandibular reconstruction. In this work we describe a process for the structured acquisition and representation of surgical knowledge for mandibular reconstruction. Based on the acquired knowledge, an $\operatorname{RDF}(\mathrm{S})$ ontology was created. The ontology is connected to the virtual planning system via a SPARQL interface. The described process of knowledge acquisition can be transferred to other surgical use cases. Furthermore, the developed ontology is characterised by a reusable and easily expandable data model.
\end{abstract}

Keywords. RDF, ontology, knowledge acquisition, CAS, mandibular reconstruction

\section{Introduction}

The integration of specific surgical knowledge into virtual planning systems plays a key role in computer-assisted surgery (CAS). This knowledge is often contained implicitly in the implemented algorithms. However, the maintainability and reusability is thus difficult to implement. A strict separation between knowledge and processing algorithms would therefore be desirable. A structured knowledge representation can also improve readability, expandability and adaptation directly by the surgeons.

Along with the Department of Oral and Maxillofacial Surgery at Heidelberg University Hospital, we are working on the development of a virtual planning system for mandibular reconstruction using autologous fibula free-flaps (FFF). Malignant neoplasms in the head and neck region are caused in $90-95 \%$ by squamous cell carcinoma [1]. The adjacent bone can be infiltrated and the mandible is most frequently affected. Ablative tumour removal with subsequent microvascular free-flap

\footnotetext{
${ }^{1}$ Corresponding Author: Niclas Hagen, E-Mail: niclas.hagen@med.uni-heidelberg.de
} 
reconstruction represents the current therapeutic gold standard. The FFF has proven to be one of the most suitable donor sites [2]. Aim is the aesthetic restoration of the face and the complete reconstruction of oral functions. Crucial functional factors are the correct occlusion between mandible and maxilla and thus a suitable basis for dental implants as well as the ability to eat in public and speak in an understandable way [2, 3]. In order to meet these high demands, exact preoperative planning plays a key role. In recent years, several virtual planning approaches, including CAD/CAM methods, have been introduced [4-6], as well as the creation of bone cutting guides for the sawing of graft segments, individually shaped plates and graft shaping guides for planning transfer [7]. In comparison to conventional planning or freehand surgery, improved clinical outcomes, shortened surgical and ischemic times as well as reduced hospital stay and complications were reported $[8,9]$. However, we are not aware of any system that provides the surgical knowledge separately from the algorithms. Therefore, we are developing a virtual planning system that enables the surgeon to automatically plan and compare different graft options based on a comprehensive knowledge base.

In this paper we describe a process for surgical knowledge acquisition in the field of mandibular reconstructions with fibula flaps and the mapping into a comprehensive ontology as well as its integration into the virtual planning system.

\section{Methods}

The ontology describes a structured mapping of the acquired surgical knowledge. Figure 1 shows the process of knowledge acquisition and its representation in the ontology. Via a query interface, the knowledge can be used within the virtual planning system for the calculation of reconstruction proposals. The planning system is based on the free open-source Medical Imaging Interaction Toolkit (MITK) of the German Cancer Research Center (DKFZ) in Heidelberg and is developed in the language $\mathrm{C}++$.

\subsection{Knowledge Acquisition}

A multi-stage process was carried out to acquire the surgical knowledge (Figure 1). First, 3D printed models of a mandible and a fibula as donor site were produced. Based on these geometric models, the possible resection and reconstruction scenarios were run through along with two senior and one junior oral and maxillofacial surgeons.

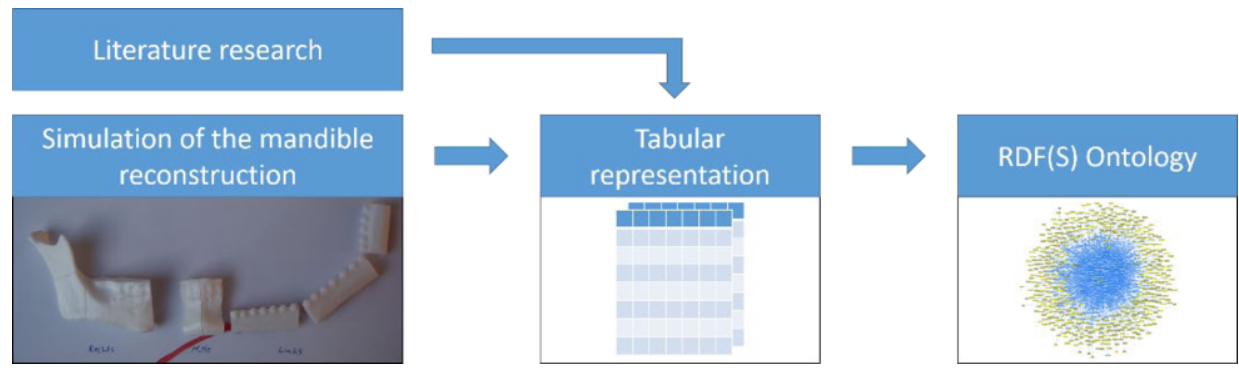

Figure 1. Knowledge acquisition and mapping into a $\mathrm{RDF}(\mathrm{S})$ ontology. Left: simulation of resection and reconstruction. Middle: Tabular representation of the acquired knowledge. Right: Knowledge mapping into $\mathrm{RDF}(\mathrm{S})$ ontology. 
To reproduce the different scenarios, the mandibular as well as the fibula model had to be subdivided into bone sections respectively bone segments. Figure 1 (left) shows the simulation of a resection in the area of the canines and a reconstruction with three fibula segments. In addition, the position of the graft blood vessel (red) as well as the position of a skin island (small spheres) for reconstruction of soft tissue defects were simulated. The scenarios were documented in tables to obtain a structured representation. Next, all scenarios were validated and checked for completeness. Then a literature search was carried out to supplement the knowledge gained. Besides the segments count, their optimal arrangement with regard to aesthetic/ functional requirements and aspects such as the position of the blood vessels, were recorded.

\subsection{Knowledge Representation and Interface to the Planning System}

With the Resource Description Framework (RDF) (https://www.w3.org/RDF/) and the vocabulary RDF Schema (RDFS) (https:/www.w3.org/TR/rdf-schema/), a lightweight ontology was formalised from the acquired knowledge. The RDF language is used to represent information on the web and is composed of various classes (resources) and their relationships. Thereby, the terminological knowledge can be described with subject-predicate-object-tiples. A subject represents a class. The predicate describes certain information of a class as well as the relationship between subject and object. For the textual representation of the ontology, the knowledge was described using the Terse RDF Triple Language (Turtle) (https://www.w3.org/TR/turtle/).

In order to use the ontology within the planning system, a corresponding interface is necessary. With the SPARQL Protocol And RDF Query Language (SPARQL) (https://www.w3.org/TR/rdf-sparql-query/), the W3C provides a standard for querying triples described in Turtle. RDF and SPARQL can be easily integrated in the MITK framework and were therefore chosen as ontology language for the planning system.

\section{Results}

To create a suitable $\mathrm{RDF}(\mathrm{S})$ data model, the definition of various classes is necessary. From the tabular listings, the five central classes OP Scenario, Anatomy, Flap, Analysis and Geometry were derived to describe reconstructive interventions. They form the core model of the ontology. In the case of mandibular reconstruction, the data model was expanded to include specific terminological and assertional knowledge.

Currently, the ontology contains 49 instances of the different mandibular reconstruction scenarios (mandible continuity resection, MCR for short) with a total of 1872 modelled relationships. The MCRs are linked with 244 different possibilities for reconstruction using fibula grafts. Furthermore, 5 anatomies (instances of Anatomy) are described. These have geometric information on anatomical landmarks, paths (e.g. central axes) and reference planes (e.g. occlusal plane). In total 38 landmarks, 8 planes and 12 paths are formalised. In addition, 24 analyses are currently described for the calculation of automated reconstruction proposals.

\subsection{Mandible Continuity Resection Scenario}

Below, the ontology structure for the MCR scenarios and its most important aspects are explained. Figure 2 shows the entity-relationship model of the MCR. 


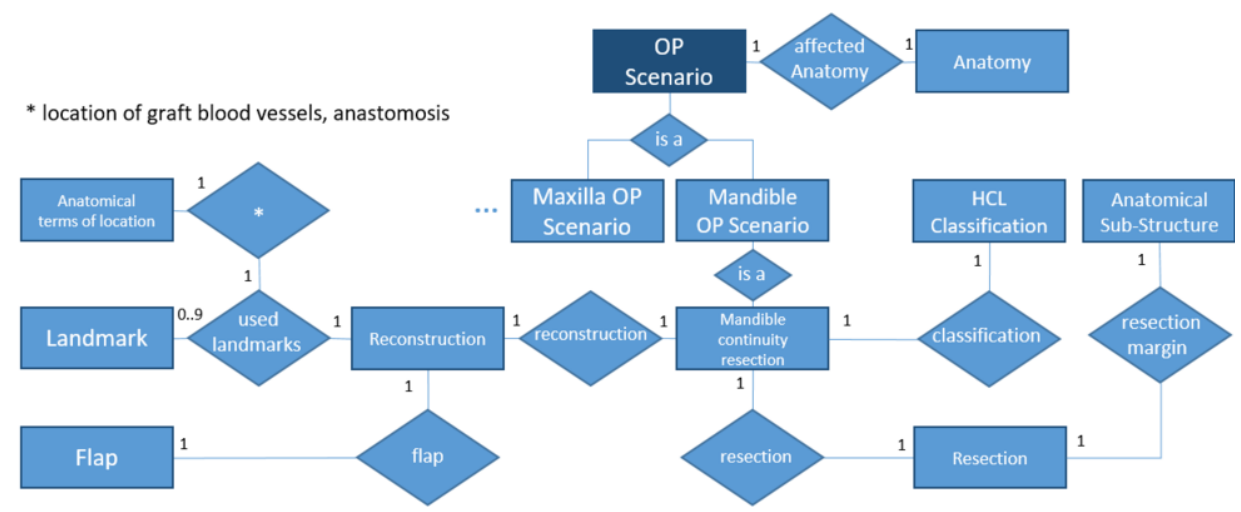

Figure 2. Entity-Relationship Model of a mandible continuity resection scenario

A surgical scenario describes a specific surgical intervention as can be seen in Figure 2. They are subdivided (e.g. Mandible OP Scenario) and related to the affected anatomy. The MCR class is associated with a resection and a set of possible reconstructions. The resection class contains information about the exact location of the cuts along the affected anatomy. Consequently, an MCR scenario is uniquely described and distinguishable. A reconstruction holds information about the used graft (right or left fibula). Furthermore, it is associated with a list of anatomical landmarks along the mandible. These landmarks serve as reference points (e.g. mandibular angle or foramen) and are used to subdivide the graft into segments. Thus, they determine the number of segments and the rough course of the graft in the recipient region. Furthermore, a reconstruction has clinical information about the graft blood vessels, their anastomosis in the recipient region and the defect extension (HCL classification).

\section{Discussion}

The developed ontology can be queried from the planning system via a SPARQL interface and successfully used for automated calculation of reconstruction proposals.

The procedure for knowledge acquisition has proven to be well suited and can be transferred to other surgical fields. The simulation of the various scenarios using 3D printed models was helpful in order to record the characteristics and to create a common basis for discussion with the surgeons. However, in order to collect surgical knowledge in a structured way with such an approach, some prior knowledge about the intervention to be described is necessary. Therefore, we divided the $3 \mathrm{D}$ printed models into valid bone sections in advance for the simulation of the different scenarios.

For structured representation of the acquired knowledge RDF(S) has proven to be a suitable language. The ontology is characterised by generic super-classes as well as reusable concepts (e.g. Anatomy). Thus, it enables knowledge modelling for further cranio-maxillofacial scenarios, such as the reconstruction of the maxilla or the midface. However, some aspects of the current data model are specifically designed for mandibular reconstruction with autologous grafts (e.g. specific landmark sets or the resection and reconstruction classes). Therefore, an extension of the existing class structures is necessary for further surgical scenarios. More, the construction of an RDF ontology is nontrivial and the textual description is at great length. This complicates the adaptation of existing models. RDFS is a lightweight ontology language. With regard 
to maintainability and validation, an upper-level ontology might be more suitable. However, the integration of such an ontology in a MITK-based system would be more elaborate.

One difficulty is the evaluation of the ontology. Thus, the knowledge can be checked for its correctness. The definition of concrete values, though, can only be assessed with the overall evaluation of the planning system. Therefore, we are planning a study on 3D printed patient cases to evaluate the reconstruction proposals of the planning system and thus the knowledge from the ontology.

\section{Conclusion}

In this paper, we demonstrate the knowledge acquisition and construction of an RDFontology for surgical knowledge representation using mandibular reconstruction as an example. Via an interface based on SPARQL, the ontology can be queried and used for the automated calculation of reconstruction proposals in the virtual planning system.

\section{Acknowledgement}

The project partners thank the Dietmar Hopp Stiftung GmbH for financially supporting our research work.

\section{References}

[1] Rasse M. Indikationen zur chirurgischen Therapie von Plattenepithelkarzinomen der Mundhöhle. Wien Med Wochenschr 2008;158:243-248.

[2] Bak M, Jacobson AS, Buchbinder D, Urken ML. Contemporary reconstruction of the mandible. Oral Oncology 2010;46:71-76.

[3] Hirsch DL, Garfein ES, Christensen AM, Weimer KA, Saddeh PB, Levine JP. Use of computer-aided design and computer-aided manufacturing to produce orthognathically ideal surgical outcomes: a paradigm shift in head and neck reconstruction. J Oral Maxillofac Surg 2009;67:2115-2122.

[4] Olsson P, Nysjö F, Rodríguez-Lorenzo A, Thor A, Hirsch J-M, Carlbom IB. Haptics-assisted Virtual Planning of Bone, Soft Tissue, and Vessels in Fibula Osteocutaneous Free Flaps. Plast Reconstr Surg Glob Open 2015;3:e479.

[5] Nakao M, Aso S, Imai Y, Ueda N, Hatanaka T, Shiba M, Kirita T, Matsuda T. Automated Planning With Multivariate Shape Descriptors for Fibular Transfer in Mandibular Reconstruction. IEEE Trans Biomed Eng 2017;64:1772-1785.

[6] Ganry L, Hersant B, Quilichini J, Leyder P, Meningaud JP. Use of the 3D surgical modelling technique with open-source software for mandibular fibula free flap reconstruction and its surgical guides. J Stomatol Oral Maxillofac Surg 2017;118:197-202.

[7] Bao T, He J, Yu C, Zhao W, Lin Y, Wang H, Liu J, Zhu H. Utilization of a pre-bent plate-positioning surgical guide system in precise mandibular reconstruction with a free fibula flap. Oral Oncology 2017;75:133-139.

[8] Wang YY, Zhang HQ, Fan S, Zhang DM, Huang ZQ, Chen WL, Ye JT, Li JS. Mandibular reconstruction with the vascularized fibula flap: comparison of virtual planning surgery and conventional surgery. Int J Oral Maxillofac Surg 2016;45:1400-1405.

[9] Tarsitano A, Battaglia S, Crimi S, Ciocca L, Scotti R, Marchetti C. Is a computer-assisted design and computer-assisted manufacturing method for mandibular reconstruction economically viable? J Craniomaxillofac Surg 2016;44:795-799. 